\title{
Nonlinear Effect of Pile-up in the Quantification of a Small Animal PET Scanner
}

\author{
Esther Vicente, Student Member, IEEE, Samuel España, Student Member, IEEE, \\ Joaquín López Herraiz, Student Member, IEEE, Elena Herranz, Student Member, IEEE, M. Desco, \\ Juan José Vaquero, Senior Member, IEEE, José Manuel Udias
}

\begin{abstract}
Accurate and reliable quantitative analysis of PET images are necessary for pre-clinical studies. To derive quantitative information from PET images, determination of the calibration curve, that is, the relationship between the pixel values in the reconstructed image and radiotracer concentrations is required. In a typical PET acquisition, several effects, such as random counts and pile-up contributions make this relationship nonlinear. We find that for PET detectors based upon relatively large PMTs like the Hamamatsu H8500, pile up may become the dominant nonlinear effect, with random contributions playing a minor role. We confirm this by means of detailed simulations of small and large cylinders in the rPET small animal scanner as well as with real acquisitions. The simulations allow us to study the impact of pile-up as a source of nonlinearity in the calibration curve of this commercially available small animal PET scanner. We compare the results obtained from images for both real and simulated data. The results show that for the activities considered in this study the quantitative results can be affected by pile-up by more than $20 \%$. We find that pile-up, which shifts counts to the center of the FOV and attenuation, which removes activity from the center of the FOV, may cancel each other for moderate activity values. This would cause quantification errors if attenuation corrections were attempted for acquisitions without pile-up corrections. The pile-up correction software improves the linearity of the calibration curve, extending the range of activity values for which a linear calibration curve can be reliably applied.
\end{abstract}

\section{INTRODUCTION}

$T^{\text {HE usefulness of small animal PET scanners in pre-clinical }}$ studies depends on accurate quantification of the data [1]. Response of lesions or tumors would only be assessed if the quantification of the reconstructed activity is stable with time and repeatable at different conditions of activity and rates. The impact of attenuation, scatter, and random counts in the

Manuscript received November 14,2008. This work was supported in part by the MEC (FPA2007-07393), CDTEAM (CENIT-Ingenio 2010) Ministerio de Industria, Spain, CPAN (Consolider-Ingenio 2010) CSPD-2007-00042 projects, and the RECAVA-RETIC network. E. Vicente acknowledges support from a CSIC-JAE predoctoral fellowship.

E. Vicente, S. España, J. L. Herraiz, E. Herranz and J. M. Udias are with the Grupo de Física Nuclear, Dpto. Física Atómica, Molecular y Nuclear, Universidad Complutense de Madrid, Spain (telephone: +34-91-394-4484, email: esther@nuclear.fis.ucm.es). E. Vicente is also with the Instituto de Estructura de la Materia, Consejo Superior de Investigaciones Cientificas (CSIC), Madrid, Spain

M. Desco and J. J. Vaquero are with the Unidad de Medicina y Cirugia Experimental, Hospital General Universitario Gregorio Marañon, Madrid, Spain (telephone: +34-91-426-5067). calibration curves is well known and a lot of different methods [2] have been proposed to correct these effects. In this work, the impact of pile-up events in the quantification of PET images is assessed. A pile-up event occurs when two or more photons strike a detector within its electronic integration time. In favorable cases, the pile-up signal has much larger amplitude than the signal from a single photon and it is rejected by energy discrimination, thus contributing only to detector dead-time. However, for actual high-resolution, high-sensitivity detector array systems, due to the use of ample energy discrimination windows to compensate for moderate energy resolution, many pile-up events are accepted as valid data. We study the impact of these accepted pile-up events in the calibration curves for 3DOSEM reconstructions, using simulations with PeneloPET Montecarlo code [3] and data acquired with the commercially available small animal PET scanner rPET [5]

\section{MATERIALS \& METHODS}

\section{A. Scanner description}

The system employed (high-resolution small-animal rPET scanner [5], SUINSA Medical Systems) has four detectors arranged in two orthogonal pairs which rotate $180^{\circ}$. Planar and tomographic images of small animals injected with positron emitting radiotracers are obtained. The detectors are $28 \times 28$ arrays of $1.5 \times 1.5 \times 12 \mathrm{~mm} 3 \mathrm{MLS}$ crystals. The array is optically coupled to a Hamamatsu H8500 flat-panel PS-PMT

The scanner has a ring diameter of $160 \mathrm{~mm}$ with effective transverse and axial field of view of $44.8 \mathrm{~mm}$. The central point sensitivity at the center of the field of view (cFOV) is $2.1 \%$ $(762.2 \mathrm{cps} / \mu \mathrm{Ci}$ ), the volumetric spatial resolution (in $\mathrm{cFOV}$ ) is $3.4 \mathrm{~mm} 3$ and the average energy resolution is $17 \%[6]$.

\section{B. Real data acquisitions}

For the calibration of the rPET scanner and the later quantification, two cylinders of different size were filled with FDG (small cylinder, $0.9 \mathrm{~cm}$ diameter, $6.5 \mathrm{~cm}$ in length; large cylinder, $5.5 \mathrm{~cm}$ diameter, $5 \mathrm{~cm}$ in length). The initial activity was known from a well-counter with accuracy of $\pm 5 \%$.

Acquisitions of 5 minutes (with different activities) were taken with both cylinders and reconstructed with a 3D-OSEM procedure [7] with different assumptions. In one of them, there was no attempt to correct for scatter or pile-up effects in the acquisition data. In the other one (improved acquisition 3DOSEM [8]), corrections for pile-up and scatter in the detector crystals are incorporated in the reconstruction algorithm 


\section{Simulation data}

In order to estimate the impact of the different effects on the calibration and the later quantification, both cylinders were simulated with PeneloPET [3],[4]. Two cases were simulated:

- With attenuation and scatter

- Without attenuation nor scatter

In both cases, sinograms of each type of coincidence in PET (trues, pile-up, randoms, scatter) were obtained and reconstructed without pile-up correction (standard 3D-OSEM)

\section{Calibration and analysis}

After reconstruction, small regions of interest (ROIs) (a few cc to prevent partial volume effects in the small cylinder) were chosen at different places in the interior of the images of both cylinders. The specific counts per cubic centimeter and second $(\mathrm{cps} / \mathrm{cc})$ measured in the ROIs were compared to the known specific activity $(\mathrm{uCi} / \mathrm{cc})$ in the inner region of both cylinders.

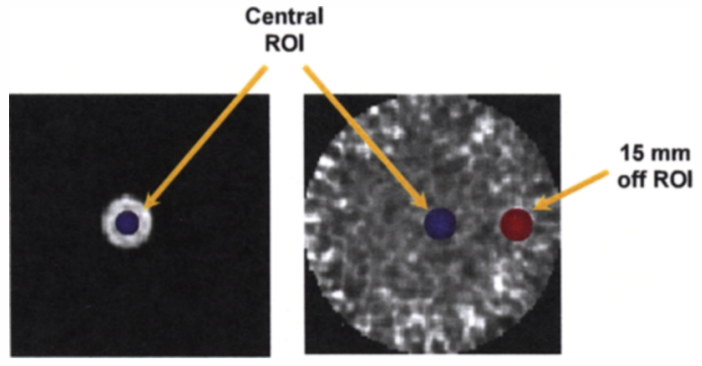

Fig. 1. ROI masks for the small cylinder (left) and the large one (right)

The data obtained with the small cylinder were fit with a linear calibration curve. A linear fit implicitly assumes that non linear effects (like random and pile-up contributions) may be neglected. The large cylinder was used to estimate the impact of pile-up in the quantification

\section{RESULTS}

In Table I the count composition for the large and small cylinder is shown. For the small cylinder, we can see that at both ends of specific activity $(2$ and $15 \mathrm{uCi} / \mathrm{cc}$, chosen because they correspond to the usual range of concentration employed for this scanner), true counts make more than $95 \%$ of the total counts recorded. Attenuation and scatter, effects whose contribution is independent on activity, also represent a very small fraction of the counts. Then, it is possible to a very good approximation to consider the activity values derived from the small cylinder as a reference to fit the calibration curve. For the large cylinder however, attenuation and scatter are sizeable effect and, for the highest activity concentration considered, half of the recorded counts experience pile-up.

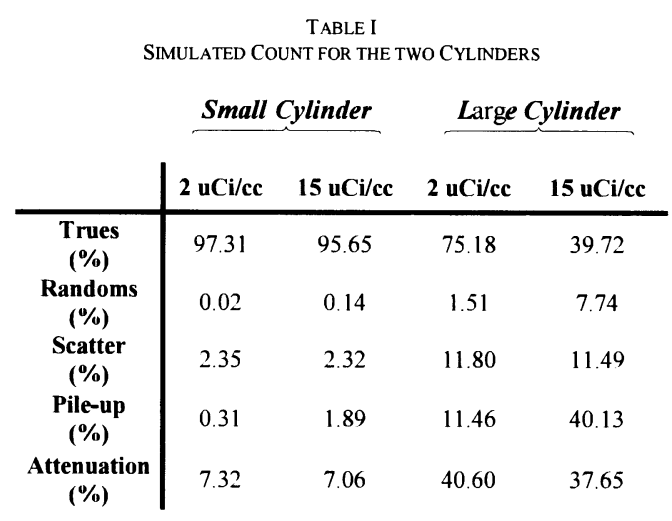

In Fig. 2 we compare the calibration curves obtained from the small cylinder with the ones obtained with the large one for both reconstruction methods, that is, standard 3D-OSEM and improved acquisition 3D-OSEM. We plot in the $\mathrm{X}$ axis the actual concentration of the cylinders, and in the $\mathrm{Y}$ axis the estimated concentration taken from the linear calibration curves. As a reference, a diagonal curve $\mathrm{Y}=\mathrm{X}$ is also shown that represents the ideal activity calibration response. In the left panel we present the results from the central ROIs in both cylinders whereas in the right panel, for the case of the large cylinder, a ROI close of its edge was taken. We can notice that the activity estimates for the small cylinder are accurate with both standard and improved methods. The differences with regards to the ideal curve are of the order of few $\%$, indicating that a linear calibration curve does a good job for the small cylinder.
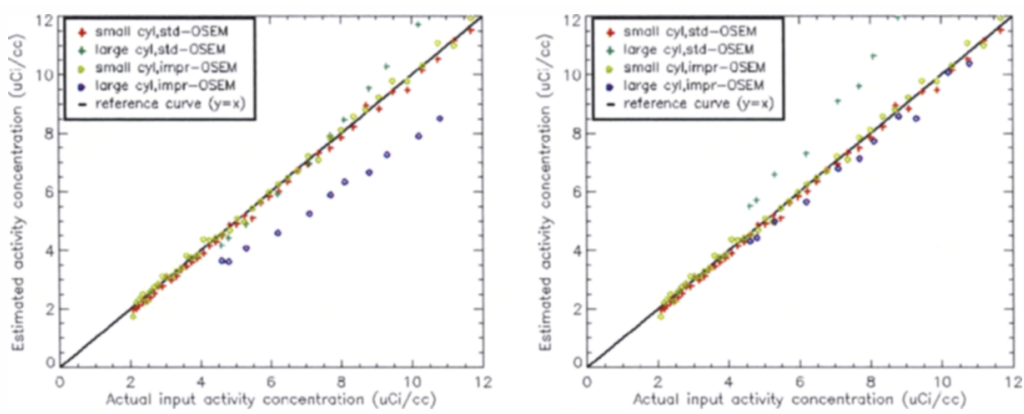

Fig. 2. Real data: Actual activities versus activities deduced from the calibration curves using the small cylinder acquisition. Left: Central ROI in the small and large cylinders. Right: Central ROI in the small cylinder, ROI near the edge in the large one ( $15 \mathrm{~mm}$-off). 
The estimates of activity from the large cylinder fall below the values they should have for the improved method in the central ROI. This is due to attenuation in the larger phantom that, according to Table I can amount up to $35 \%$. Indeed, the estimated activity in the center of the large cylinder falls below the actual activity by near $35 \%$. However, a good linearity is still seen for these large cylinder data. The standard OSEM seems to do a good job for low activity concentrations but it overestimates activity at the high end of the curve. This is due to the compensation of the lost counts (attenuation) with pile-up ones. This compensation cannot happen for the improved OSEM.
We can see in the right panel that for the external ROIs, where attenuation is less important, the improved OSEM is near the ideal curve while the standard OSEM overestimates the activity concentration and the slope is clearly different and nonlinear. If attenuation correction were introduced into the reconstructions, the improved OSEM reconstruction will produce good activity estimates, while the standard acquisitions will yield erroneous activity estimates, with an increasing error for larger activities. In conclusion, pile-up suppression will improve the quantification of PET images of tomographs like the rPET scanner

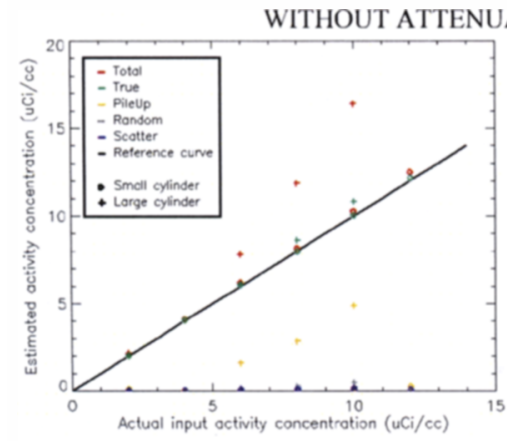

(A)

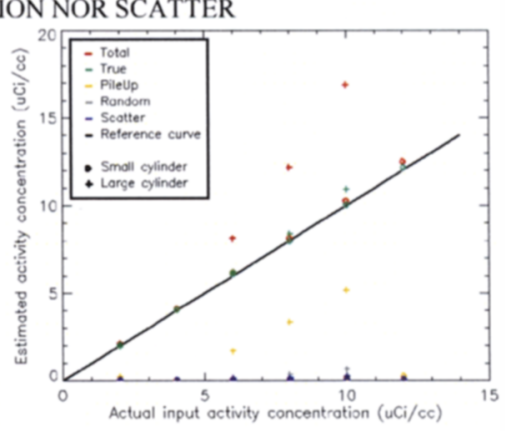

(B)

WITH ATTENUATION AND SCATTER

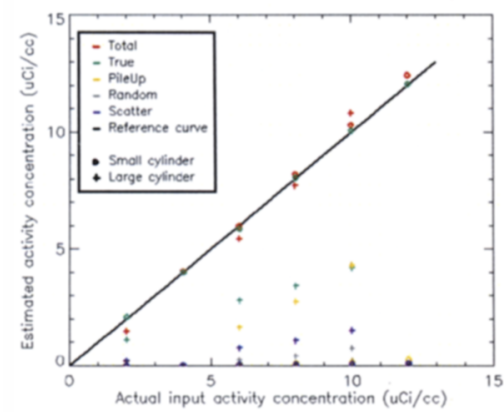

(C)

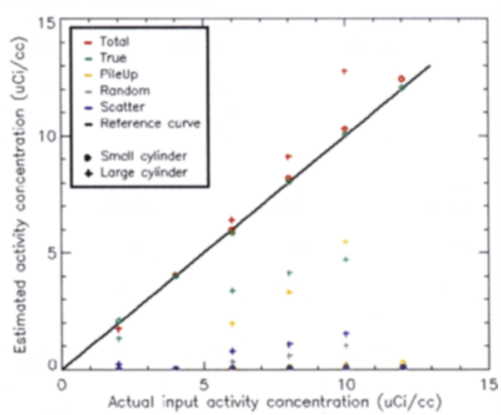

(D)

Fig. 3. Simulated data: Actual activities versus activities deduced from the calibration curves using the small cylinder. The contribution of the different types of coincidences is also shown. In the top panels data withou attenuation nor scatter (A) and (C): Central ROI in the small and large cylinders. (B) and (D): Central ROI in the small cylinder, $\mathrm{ROI}$ near the edge in the large one (15 mm-off)

This behavior is reproduced in the simulated data (reconstructed with standard 3D-OSEM, without pile-up correction) where we can see that if there is no attenuation (it means, the attenuation has been corrected) overestimation takes place with the large cylinder in central and external ROIs (Fig. 3, [A] and [B]). However, when the attenuation is not corrected (Fig. 3, [C] and [D]), pile-up counts in the center of the image compensate the losses due to the attenuation but in the edges, where the mean attenuation is much lower, overestimation appears again.
In Fig. 4 and Fig. 5 average line profiles along the large cylinder image are presented for real and simulated studies The different activities have been rescaled to $1 \mathrm{uCi} / \mathrm{cc}$ to study how suitable is the quantification in each case. The profiles of the different type of coincidences are shown for the simulated results (Fig. 5). 


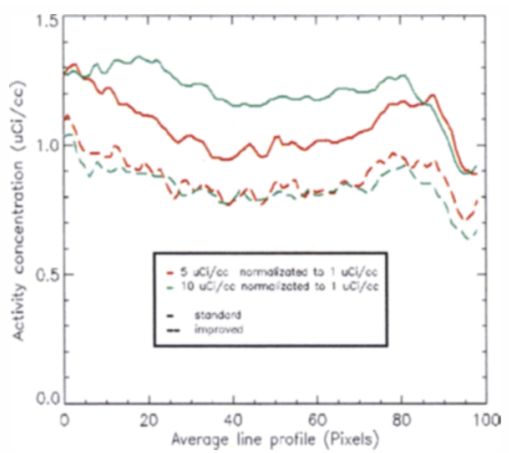

Fig. 4. Real data: Average line profiles of the real large cylinder images for two activities. Profiles have been rescaled to $1 \mathrm{uCi} / \mathrm{cc}$ results.

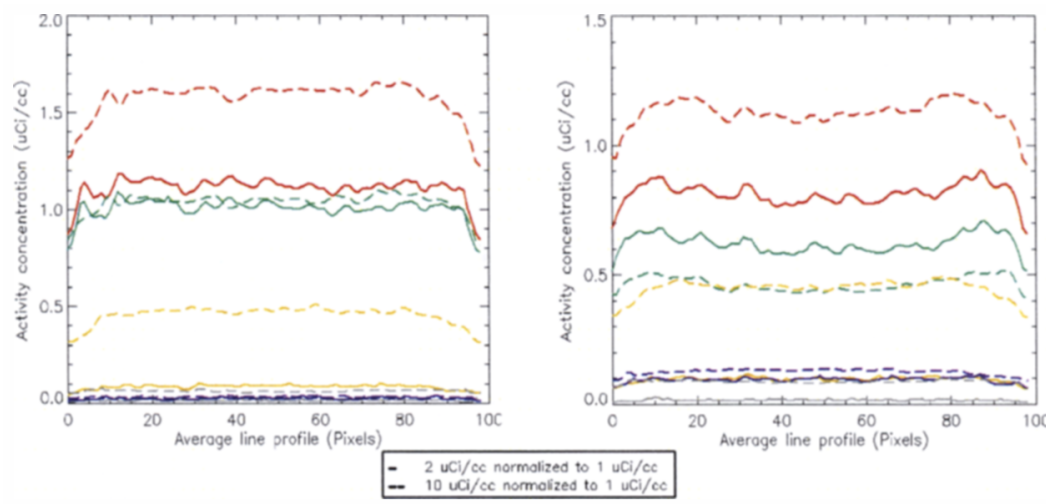

Fig. 5. Simulated data: Average line profiles of the simulated large cylinder images for two activities rescaled to $1 \mathrm{uCi} / \mathrm{cc}$. Profiles of the different types of coincidences are also shown.

\section{CONCLUSIONS}

I) Calibration curves have to be obtained from acquisitions free of undesired contributions such as pileup. Using a small volume phantom is a suitable choice

II) Quantification from the images obtained with the improved 3D-OSEM method is independent of the activity. This shows that the method corrects pile-up coincidences

III) For accurate quantification, both pile-up and attenuation corrections are needed. These effects somewhat cancel each other. If correcting only one of them, very inaccurate results can arise

\section{ACKNOWLEDGMENT}

Part of the computations of this work were done at the "High Capacity Cluster for Physical Techniques" of the Faculty for Physical
Sciences of the UCM, funded in part by the UE under the FEDER program and in part by the UCM.

\section{REFERENCES}

[1] Irene Buvat. Quantification in emission tomography: Challenges, solutions and performance. NIM A 571 (2007)

[2] Dale L. Bailey, David W. Townsend, Peter E. Valk, Michael N. Maisey (Editor). Positron Emission Tomography: Basic Sciences Editorial: Springer, London 2003

[3] S. Espana, J. L. Herraiz, Vicente, E., J. J. Vaquero, M. Desco, and J. M. Udias, "PeneloPET: A MonteCarlo PET simulation tool based on PENELOPE," presented at IEEE Nuclear Science Symposium and Medical Imaging Conference, 2006.

[4] S. España, J. L. Herraiz, Vicente, E., Herranz, E.. J. J. Vaquero, M. Desco, and J. M. Udias; "Validation of PeneloPET against two small animal PET scanners" ,IEEE NSS-MIC Conf. Rec 2007, Vol 4, pp. 2597-2601.

[5] J. J. Vaquero, E. Lage, L. Ricon, M. Abella, E. Vicente, and M Desco. rPET Detector Design and Data Processing. 2005 IEEE Nuclear Science Symposium Conference Record, 2005; pp. 28852889 
[6] Vaquero, J.J. Lage, E. Redondo, S. Abella, M. Pascau, J. Sanchez, J. Vicente, E. Soto-Montenegro, M.L. Desco, M. Co-planar PETCT for small animal imaging. IEEE Nuclear Science Symposium Conference Record, 2005; pp. 1748-1751.

[7] J. L. Herraiz, S. Espana, J. J. Vaquero, M. Desco, and J. M. Udias, FIRST: Fast Iterative Reconstruction Software for (PET) tomography. Phys. Med. Biol., vol. 51, pp. 4547, 2006. Fan-Beam Collimators," Nuclear Science, IEEE Transactions on, vol. 50, 2003 pp. 398-404.

[8] S. España, J. L. Herraiz, E. Vicente, E. Herranz, J. J. Vaquero, M Desco, J. M. Udias. Improved image reconstruction in small animal PET using a priori estimation of single-pixel events. 2007 IEEE Nuclear Science Symposium Conference Record, 3876-3880, 2007 